row scanners are mandatory. The evaluation algorithm follows the recommendation of the National Comprehensive Cancer Network (NCCN Guideline) which specifies interventions according to nodule size. Suspicious findings lead to individual assessment by a pulmonologist and could imply CT control after several weeks, PET imaging, or immediate biopsy. Clinical workup and treatment for malignancies follow the respective guidelines.

Conclusion In Germany, formerly asbestos-exposed insured individuals have the statutory right to receive 'follow-up occupational medical examinations' (secondary prevention). Due to the results of the NLST-Study, the DGUV decided to offer an annual low-dose CT to a highly selected population of former asbestos-exposed workers and workers with asbestos-induced recognised occupational disease. Hereby, we present results of this early detection program.

\section{ASBESTOS RELATED OCCUPATIONAL DISEASES - LEGISLATIVE CHANGES AND INCIDENCE DIFFERENCES IN CROATIA}

Ina Kardoš* ${ }^{*}$ Marina Milaković. Croatian Institute for Health Protection and Safety at Work, Zagreb, Croatia

\subsection{6/oemed-2018-ICOHabstracts. 1291}

Introduction The aim of this study was to identify differences in the occurrence of asbestos-related occupational diseases in relation to the changes in Croatian legislation regarding health protection, surveillance and compensation entitlement due to occupational exposure to asbestos. Aforementioned changes occurred in the period between 2007 and 2008 and are considered as a significant step forward in registering asbestosrelated occupational diseases.

Methods Annual epidemiological and demographic data on newly diagnosed patients with asbestos related diseases have been retrieved from Register of Asbestos Related Occupational Diseases of the Croatian Institute for Health Protection and Safety at Work for the period between 2009 and 2016. Annual Croatian Health Service Yearbooks of the Croatian National Institute for Public Health have been used for obtaining data between 2000 and 2008. Descriptive statistical analysis has been performed.

Results Between 2009 and 20161356 asbestos-related occupational diseases have been diagnosed with peak occurrence in 2011, following: 52\% pleural plaques with asbestosis $(n=705)$, $22.27 \%$ pleural plaques $(n=302), 20.13 \%$ pulmonary asbestosis $(n=273), 4.28 \%$ mesothelioma $(n=58)$ and $1.33 \%$ other respiratory malignant neoplasms $(n=18)$. Between 2000 and 2008104 asbestos-related occupational diseases have been reported (nearly 13 times less compared to the post-legislative period).

Discussion Changes in legislation have, undoubtedly, led to significant differences in the occurrence of occupational asbestosrelated diseases. Reasons for such differences might be partially due to workers' inclination to compensation and indemnification after being diagnosed with occupational disease. However, a relatively late onset of a complete ban of asbestos-engaged work in Croatia is also considered to be a major contributing factor for present results. Considering relatively late peak of occurrence in 2011, it is fair to assume that a significant number of asbestos-related diseases are yet to be reported. Hence, a further need for continuous health surveillance of workers exposed to asbestos cannot be accentuated enough.

\section{SILICA EXPOSURE AND WORK-RELATEDNESS EVALUATION FOR OCCUPATIONAL CANCER IN KOREA}

${ }^{1}$ Hyoung-Ryoul Kim*, ${ }^{2}$ Boowook Kim, ${ }^{1}$ Bum Seak Jo, ${ }^{1} \mathrm{Ji}$-Won Lee. ${ }^{1}$ Dept Occup and Environ Med, College of Medicine, the Catholic University of Korea, Seoul, South Korea; ${ }^{2}$ Occupational Lung Disease Institute, Korea Workers' Compensation and Welfare Service, Incheon, Korea

\subsection{6/oemed-2018-ICOHabstracts. 1292}

Introduction Crystalline silica has been classified as a definite carcinogen (Group 1) causing lung cancer by the International Agency for Research on Cancer (IARC). In Korea, crystalline silica has been the most common causal agent for workers to apply to the Korea Workers' Compensation and Welfare Service (KWCWS).

Methods We used KWCWS data to evaluate workers' crystalline silica exposure levels according to their occupations and industries, and reviewed research papers describing the doseresponse relationship between cumulative exposure levels and lung cancer incidence. In addition, we reviewed lung cancer cases accepted by the KWCWS, and suggest new criteria for defining occupational cancer caused by crystalline silica in Korea.

Results A review of 120 cases of occupational lung cancer confirmed through an epidemiological survey of the KWCWS since 2007 revealed that 45 cases (37.5\%) involved miners. Most of the coincidental exposures (72.5\%) involved asbestos, diesel exhaust particles, radon, hexavalent chromium, and so on. From the carcinogenic exposure to the onset of the disease, there was no case of less than 10 years, and the lag time was more than 40 years in $40 \%$ of the cases. Among the patients whose cases were approved, 19.2\% had pneumoconiosis, while many cases were approved without pneumoconiosis. A high level of exposure was found in the construction industry, and significant exposures were also confirmed among miners and foundry workers. Stone quarrying and stone laying were also found to expose workers to high concentrations of crystalline silica.

Discussion Rather than confining to miners, we propose recognising occupational lung cancer whenever workers with pneumoconiosis develop lung cancer, regardless of their industry. Coincidental exposure and lag time should also be considered in evaluations of work-relatedness.

\section{AN ANALYSIS OF LATENT PERIOD AND SURVIVAL FACTORS OF KOREAN PATIENTS WITH MALIGNANT MESOTHELIOMA}

${ }^{1}$ Sung Soo Lee*, ${ }^{1}$ Eun Chul Jang, ${ }^{2}$ Dong \| kim, ${ }^{3}$ Dongmuk Kang, ${ }^{1}$ Yong Jin Lee. ${ }^{1}$ Soonchunhang University, Chunan, Korea; ${ }^{2}$ Seonam University, Seoul, Korea; ${ }^{3}$ Pusan University, Pusan, Korea

\subsection{6/oemed-2018-ICOHabstracts. 1293}

Introduction Malignant mesothelioma is an aggressive tumour occurring in mesothelioma cells of pleura and peritoneum. About $80 \%$ of malignant mesothelioma cases are known to be caused by asbestos. Malignant mesothelioma is known to have a very poor prognosis with an average survival period of about 12 months. The number of patients of malignant 
mesothelioma in Korea is smaller than that of some developed countries. However, mesothelioma has increased greatly in recent years in Korea, and it is expected to increase continuously considering asbestos consumption, as it happened in other countries which used large amounts of asbestos. It is important to investigate the epidemiologic characteristics and prognostic factors of malignant mesothelioma in Korea.

Methods A total of 728 patients who received asbestos-related relief from malignant mesothelioma by 2014 were included in the study. In $2015,150(20.6 \%)$ out of 728 people were surveyed. Interviews were conducted with structured questionnaires for patients with malignant mesothelioma and their families. The age, sex, surgical status, route of exposure, and age at diagnosis of malignant mesothelioma patients were analysed using the proportional hazard model of Cox.

Results Ninety eight (65.3\%) males and 52 females (34.7\%) had malignant mesothelioma according to sex. In the case of mesothelioma according to age, 49 cases (32.7\%) were the highest in above $70 \mathrm{~s}, 42$ cases $(28.0 \%)$ in the $60 \mathrm{~s}, 40$ cases $(26.7 \%)$ in the $50 \mathrm{~s}$, Followed by below 49 to $19(12.7 \%)$. In this study, asbestos exposure source of subjects was $40.7 \%$ for occupational factors and $56.0 \%$ for environmental factors, which was higher than 59\% of Kim, et al. 's (2009) study.

The latent period was $35.0 \pm 15.8$ years, which was mostly latent period of 30 years or more. And 39.1 \pm 15.1 years in the occupational asbestos exposure group and 32.2 \pm 15.7 years in the non occupational asbestos exposure group. The mean survival duration after diagnosis of mesothelioma was 19.9 \pm 27.2 months. Mean occupational exposure was $15.8 \pm 21.3$ months in occupational asbestos exposure group and 22.8 \pm 30.5 months in non occupational asbestos exposure group. Gender, exposure type, and age at diagnosis did not significantly affect the risk of malignant mesothelioma death. The risk of death was 2.20 times (95\% CI: 1.15 3.56) higher in the pleura than in the other sites of malignant mesothelioma. Also, according to the received surgery, the number of patients who underwent surgery was lower by 0.52 times (95\% CI: $0.33 \sim 0.81$ ) than those without surgery.

Conclusion This study revealed that the site of onset and surgical treatment had an effect on the risk of death in patients with malignant mesothelioma. It is necessary to develop a new treatment and compensation method for malignant mesothelioma which is expected to increase rapidly in the future and to plan ways to minimise exposure to future asbestos.

\section{SURVIVAL ANALYSIS OF MALIGNANT PLEURAL MESOTHELIOMA IN MEXICAN WORKERS}

${ }^{1} \mathrm{C}$ Madrigal ${ }^{*},{ }^{1} \mathrm{G}$ Aguilar, ${ }^{1} \mathrm{CA}$ Juárez, ${ }^{1} \mathrm{O}$ Trujillo, ${ }^{2} \mathrm{C}$ Jiménez, 'LA Trejo, ${ }^{1} \mathrm{D}$ Fierro, ${ }^{3}$ ES Calderón. 'Occupational Health Research Unit, IMSS, Mexico City, Mexico; ${ }^{2}$ Hospital of Traumatology, Orthopaedics and Rehabilitation 'Dr. Victorio de la Fuente Narvaez'. IMSS. Mexico City, Mexico; ${ }^{3}$ Department of Toxicology. Research Centre for Advanced Studies, CINVESTAV. Mexico City, Mexico

\subsection{6/oemed-2018-ICOHabstracts. 1294}

Introduction Malignant Pleural Mesothelioma (MMP) is a neoplasm with high mortality caused by exposure to asbestos. Patients with MMP have a short survival with a median of 9 months (4-18 months); the worldwide increase in MMP incidence and mortality is more than 120, 000 cases. In Mexico, it is estimated 500 cases of MMP per year; however, there are no survival studies for this cancer. The aim of this study was to perform a case survival analysis with MMP and to identify the factors related to it.

Methods From a case study (MMP) and controls conducted from 2011-2016 in 3 hospitals in Mexico City, performed a survival analysis with the Cox model to obtain the Hazard Ratio (HR) with MMP, tumour stage, age, sex and history of occupational exposure.

Results Of the 187 cases of MMP incorporated, there was a median survival of 480 days (IQR: 239-750). A Cox model was performed obtaining an Hazard Ratio by age of 1.02 (95\% CI: 1.006 to 1.04 ), by asbestos occupational exposure of 1.84 (95\% CI: 0.95 to 3.59 ) and stage IV of $1.95(95 \%$ CI: 1.34 to 2.85 ) adjusted by sex.

Discussion We observed that survival results are similar to those reported in the literature, that the risk of dying from MMP increases with age, occupational exposure to asbestos and tumour stage. MMP is diagnosed in advanced stages, thus survival is short, so that it is fundamental to continue the research of molecular markers for early diagnosis and to offer a timely treatment to increase survival and quality of life of patients.

\section{TUBERCULOSIS AND SILICOSIS DIAGNOSTIC CRISIS - A ZIMBABWE CASE SERIES REPORT}

${ }^{1,2}$ D Moyo*. 'Baines Occupational and Travel Health Centre, Harare, Zimbabwe; ${ }^{2}$ Midlands State University, Gweru, Zimbabwe

\subsection{6/oemed-2018-ICOHabstracts. 1295}

Introduction Zimbabwe has a long history of extensive hard rock mining and it is likely that the occupational lung disease (OLD) burden, for conditions such as silicosis and silico-tuberculosis, is huge. Poor access by miners to occupational health and safety services and lack of occupational health $(\mathrm{OH})$ diagnostic skills against a background of a high tuberculosis burden in developing countries such as Zimbabwe presents a significant challenge in the diagnosis of pulmonary tuberculosis (PTB) and or silicosis amongst miners and ex miners.

Methods This is a case series study of five ex- gold and quarry miners who worked in different mines in Zimbabwe. This study reviewed the diagnosis and management of tuberculosis and silicosis among the five ex-miners.

Results Despite a typical clinical presentation and radiological findings of silicosis, all the cases were misdiagnosed and treated for PTB. They had all been treated for PTB with two of them having been treated twice. In all the five cases, sputum for alcohol and acid fast bacilli (AAFBs) was negative with two of the cases having tested AAFBs negative on two different occasions. All the cases had had chest $\mathrm{x}$ rays that revealed a reticulonodular pattern. The mean occupational exposure period to silica containing dust was 9.4 years with a range of 3 months to 15 years. The mean period from clinical presentation to diagnosis of silicosis was 18 months.

Conclusions Silicosis and tuberculosis have similar presentation and lack of $\mathrm{OH}$ diagnostic skills can lead to unnecessary PTB treatment and delayed diagnosis of silicosis and an increased risk of tuberculosis disease. Poor $\mathrm{OH}$ diagnostic skills among clinicians in developing countries such as Zimbabwe can lead to delayed diagnosis of OLDs. Clinicians should be equipped with fundamental $\mathrm{OH}$ training and diagnostic skills in order to be able to diagnose OLDs and PTB. 